\title{
Simplification of the forced response of mistuned bladed disks using multiple scales techniques
}

\author{
Juan A. Martín • Carlos Martel
}

Received: 27 December 2020 / Accepted: 19 March 2021

(1) The Author(s), under exclusive licence to Springer Nature B.V. 2021

\begin{abstract}
Mistuning can produce a considerable increase of the vibratory forced response of the blades compared with that of the tuned bladed disk. This situation can lead to high cycle fatigue failure, and, therefore, it is of crucial importance for the prediction of the safe operation limit of the bladed disk. Nonlinear friction forces determine the final vibration amplitude of the blades, and, because of mistuning, the complete bladed disk has to be considered, increasing considerably the difficulty of the problem. For this reason, we consider the application of asymptotic multiple scale techniques to derive simplified models that give the final vibration states at a very low CPU cost. This asymptotic method is based on the idea that all significant effects (forcing, nonlinear friction damping, and mistuning) produce, in most practical situations, just small corrections of the tuned blade elastic oscillation. These small effects develop in a much longer time scale than that associated with the natural elastic frequency of the tuned system. The reduced models produced by this asymptotic methodology retain only the slow time dynamics, filtering out the fast scale oscillation. In this paper, the bladed disk is described using a mass-spring system with nonlinear friction and with an external forcing acting on a blade-dominated modal family where all modes have very similar vibration frequencies. The derivation of the asymptotic model from
\end{abstract}

the mass-spring system is explained in detail, and validated against the results from the original mass-spring model.

Keywords Asymptotic method - Blade vibration . Nonlinear friction - Turbomachinery

\section{Introduction}

The ever increasing demand for higher efficiency in modern turbomachinery moves the designs towards higher loadings and thinner blades. This makes the bladed disks more susceptible to experience aeromechanic vibrations. The sources of blade vibration energy (flutter, forced response,...) must be counterbalanced by the dissipation produced by the nonlinear friction forces at the interfaces with contact between blade and disk, and this balance determines the final vibration amplitude of the blade. The amplitude of the blade vibration is of extreme practical importance in order to estimate the safe blade operative life, as it constitutes a key information for the correct estimation of the high cycle fatigue (HCF) level.

In a turbomachine, the upstream stator generates a pulsating aerodynamic force over the rotor, that takes the form of a travelling wave (in the rotor frame) and can be assumed to be approximately composed of a single harmonic in time. The vibration of the blade and the disk can be accurately modelled using linear elasticity, but the friction forces at the contact interfaces follow 
a nonlinear model $[12,13]$. The vibration response of the blades is no longer composed of a single harmonic because the nonlinearity generates multiple harmonics that necessarily have to be taken into account.

The harmonic balance method (HBM) is normally used for the calculation of the blade vibration in the frequency plane (see, fi.i., $[5,10,11]$ ). The HBM requires to solve a large system of nonlinear algebraic equations. The number of equations grows proportionally to the number of harmonics that are included in the description, and, in order to properly represent the highly nonlinear behaviour of the friction effects, a considerable number of harmonics has to be kept, despite of the fact that only a small fraction of the total number of degrees of freedom includes nonlinear terms.

In the case of a tuned rotor, the HBM calculation is reduced to a single sector with appropriate phase lag boundary conditions. When mistuning is taken into account, the cyclic symmetry of the problem is lost, and consequently the complete bladed disk has to be considered. The computational cost of the mistuned case is thus dramatically increased, making it imperative to look for methods that allow for the reduction of the problem complexity, keeping a satisfactory level of accuracy.

In previous works, the multiple scale method has been successfilly applied to the study of the vibration amplitude saturation by nonlinear friction forces in aerodynamically unstable rotors, as can be found in [8]. It has been also employed to the calculation of the friction effects on the forced response of a mistuned bladed disk, for the case of exciting an isolated mode [7]. In these latter works, only two travelling waves (TW) are exhibited in the response of the system.

In the present paper, the multiple scale method $[1,4]$ is applied to derive a simplified model for a mistuned bladed disk, in the more complex situation of forcing a mode that belongs to a blade-dominated modal family. All TW modes in the family have very similar frequencies and, accordingly, the mistuning coupling effect gives rise to a vibration response that can contain many TWs. Therefore, the idea of this paper is to present the derivation of this simplified asymptotic model, and to check its accuracy. To this end, the mistuned bladed disk is represented by a mass-spring model with 2 degrees of freedom (DOF) per sector (blade vibration and friction displacement). From this mass-spring model, a very reduced asymptotic model is derived for the tuned and the mistuned cases, and the

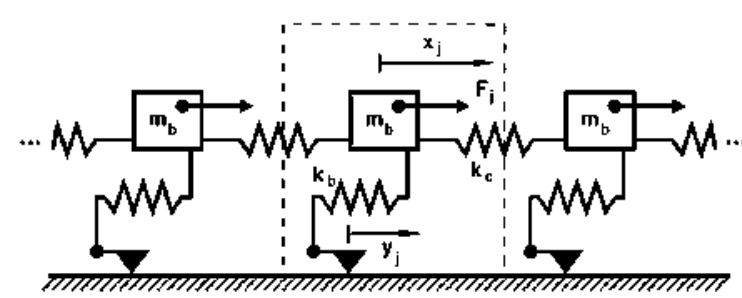

Fig. 1 Mass-spring model

results from the simplified model are compared with those from the original mass-spring model.

\section{Mass-spring simplified model of a bladed disk}

The formulation here employed follows closely the one presented in [7]. The mass-spring model sketched in Fig. 1 is a simplified description of the forcing of a bladed disk with mistuning and nonlinear friction effects included. It contains a total of $N$ sectors, with 2 DOF per sector: the blade displacement, $x_{j}$, and the microslip friction displacement at the bladed disk contact interface, $y_{j}$.

The corresponding equations of motion for the tuned case can be written as:

$$
\begin{aligned}
& m_{b} \frac{d^{2} x_{j}}{d t^{2}}+k_{b}\left(x_{j}-y_{j}\right)+k_{c}\left(2 x_{j}-x_{j+1}-x_{j-1}\right) \\
& +c \frac{d x_{j}}{d t}=F \mathrm{e}^{i \omega t+\mathrm{i} E O \frac{2 \pi}{N} j}+c . c . \\
& m_{f} \frac{d^{2} y_{j}}{d t^{2}}+k_{b}\left(y_{j}-x_{j}\right)+F_{j}\left(y_{j}\right)=0 \text { for } j=1, \ldots, N
\end{aligned}
$$

where $m_{b}$ is the mass of the blade, $k_{b}$ is the blade stiffness, $c$ is a small material damping, and $k_{c}$ represents the stiffness of the coupling with the adjacent blades (i.e., the effect of the coupling of the blades through the disk). The external forcing is represented as a TW with amplitude $F$, engine order $E O$, and frequency $\omega$, which accounts for the effect of the wakes of the upstream row. It is convenient to write the forcing using complex notation; c.c. means "complex conjugate" and the resulting final expression for the forcing is a real TW given by $2 F \cos \left(\omega t+E O \frac{2 \pi}{N} j\right)$. The friction degree of freedom has a small mass, $m_{f}$, and $F_{f}\left(y_{j}\right)$ represents the nonlinear friction force that depends only on the friction displacement $y_{j}$.

For the friction force $F_{f}$, the Olotsson microslip model [9] is employed. The magnitude of the tric- 


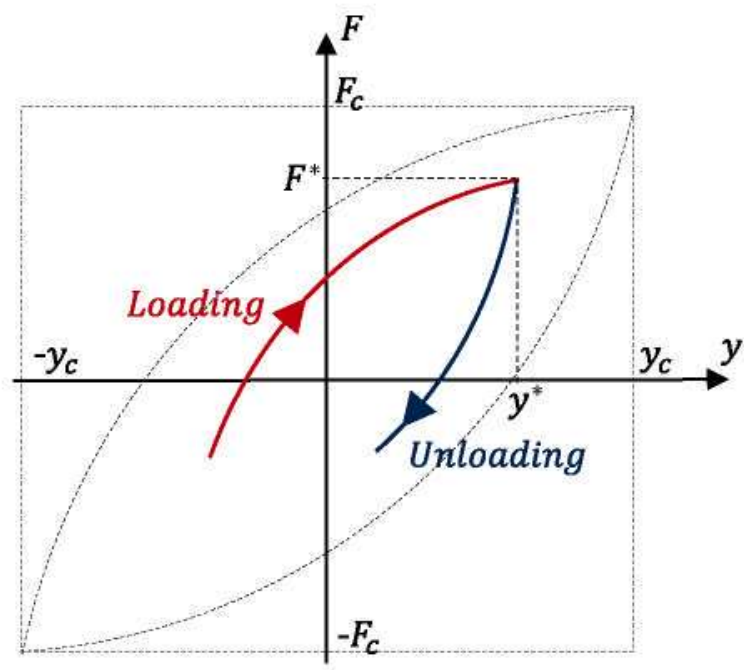

Fig. 2 Olofsson friction force. Dotted lines mark the boundaries of the microslip regime

tion force for a cycle of loading and unloading can be expressed as:

$F_{f}(y)=F^{*} \pm F(y)$

with $F^{*}$ taken from the preceding loading-unloading switch point, the + sign is used for a loading case, and - for the unloading, as represented in Fig. 2. The variation of the friction force $F(y)$ is given by:

$F(y)=2 F_{c}\left(1-\left(1-\frac{\left|y-y^{*}\right|}{2 y_{c}}\right)^{\frac{5}{2}}\right)$

where $y^{*}$ denotes the displacement at the previous loading-unloading switch point. The microslip regime is delimited by the microslip characteristic force $F_{C}$ and displacement $y_{c}$. There is, therefore, a maximum increment of friction force and displacement of magnitude $2 F_{C}$ and $2 y_{c}$ inside the microslip regime. For higher values of the friction displacement the system would enter the macroslip regime that requires a different expression for the friction force. The analysis in this paper is restricted to the microslip regime, which is a representative situation in some Low Pressure Turbine situations (see, e.g., [2]).

The Olofsson nonlinear friction force model involves two effects: the first one is the characteristic stiffness measured as $F_{c} / y_{c}$, i.e., the slope of the straight line connecting the origin with the point $\left(y_{c}, F_{c}\right)$ in Fig. 2 (that is, an equivalent linear spring for the stiffness effect of the friction force); and the second one, the hysteresis effect that results in a damping action as a consequence of the different force values involved in the loading and unloading phases.

The system (1) is made nondimensional in order to easily compare the relative importance of the different effects. Time is scaled with the blade alone vibration frequency:

$T=t \omega_{b}=t \sqrt{\frac{k_{b}}{m_{b}}}$,

the friction displacement and force are measured with respect to their characteristic values

$\tilde{y}=y / y_{C}, \quad \tilde{F}_{f}(\tilde{y})=F_{f}(y) / F_{C}$,

the characteristic value of the blade displacement, $x_{c}$, is obtained from the balance of the friction force with the force exerted by the blade at the friction contact $\left(F_{c}=k_{b} x_{c}\right)$

$\tilde{x}=x / x_{c}=x /\left(F_{c} / k_{b}\right)$,

the forcing is scaled with the forces produced by the friction displacement on the blade

$f=F /\left(k_{b} y_{c}\right)$,

and, finally, the forcing frequency is also scaled with the blade alone vibration frequency

$\tilde{\omega}=\omega / \omega_{b}$.

The resulting nondimensional system of equations takes the form

$$
\begin{aligned}
& \frac{d^{2} \tilde{x}_{j}}{d T^{2}}+\left(\tilde{x}_{j}-\theta \tilde{y}_{j}\right)+\theta k\left(2 \tilde{x}_{j}-\tilde{x}_{j+1}-\tilde{x}_{j-1}\right) \\
& +\theta c \frac{d \tilde{x}_{j}}{d T}=\theta f \mathrm{e}^{i \tilde{\omega} T+\mathrm{i} E O \frac{2 \pi}{N} j}+\text { c.c. } \\
& \theta \gamma \frac{d^{2} \tilde{y}_{j}}{d T^{2}}+\left(\theta \tilde{y}_{j}-\tilde{x}_{j}\right)+\tilde{F}_{f}\left(\tilde{y}_{j}\right)=0 \text { for } j=1, \ldots, N
\end{aligned}
$$

There are three more nondimensional parameters in the system above:

$\gamma=\frac{m_{f}}{m_{b}}$,

which is the ratio of the masses of the friction DOF and blade DOF,

$\theta=\frac{y_{c}}{x_{c}}=\frac{k_{b}}{\left(F_{c} / y_{c}\right)}$

which is the friction to blade characteristic displacement ratio, and is typically very small, $\theta \ll 1$, and the 
relation between blade and blade-to-blade stiffness is denoted

$\frac{k_{c}}{k_{b}}=\theta k$

where the parameter $k \sim 1$.

The case analyzed in this paper corresponds to a nearly flat blade-dominated modal tamily. Therefore, the ratio between blade and coupling stiffness is also taken to be very small, $\theta k \ll 1$. It represents a small blade coupling effect induced by the disk.

The limit $\theta \ll 1$ indicates that the elastic forces are dominant in the dynamics of the system, and the effect of friction is very small. The magnitude of the forcing is assumed to be of order $\theta$ in the nondimensional system (9). This is due to the dissipation induced by the friction forces, that has to balance with the energy pumped into the system by the external forcing. The growth of the vibration amplitude is therefore saturated in the final vibration state.

In the next sections, the system of equations (9) will be analyzed using a multiple scales method based on the small parameter $\theta$ (see $[1,4]$ for a more complete description this methodology). This asymptotic procedure will provide a very simplified description for the dynamics of the mass-spring system (9), for the case of both tuned and mistuned configurations.

\section{Tuned response}

The common situation where the friction effects are much smaller than the elastic forces is represented as the asymptotic limit of very small $\theta$. The blade elastic forces are dominant, and, in first approximation, the response of the system is given by the elastic blade vibration modes without friction and forcing. The friction effect is then computed as a small correction of the displacement at the contact interfaces, generating a small adjustment of the purely elastic vibration characteristics. A large number of elastic oscillation periods is required to see the action of the small effects associated with the forcing and the friction, whose balance selects the resulting final value of the blade vibration amplitude.

The multiple scales method (see $[1,4]$ ) is applied below to approximate the dynamics of the system (9), which, due to the existence of a small parameter, exhibits two very disparate time scales: fast (elastic) and slow (disk coupling, forcing and friction). The key idea of the multiple scales method is to introduce two time scale variables (fast and slow) that are handled as if they were independent. The solution is then expanded in powers of the small parameter, and the fast time scale is filtered out to obtain the slow time evolution of the system. This is achieved by making use of the so-called solvability conditions, that eliminate the possibility of unbounded behavior in the fast time scale (see [7] for a similar asymptotic derivation in a much simpler case with only two TWs involved in the response).

The solution of system (9) is first expanded as

$\tilde{x}_{j}=\tilde{x}_{j}^{0}(T, \tau)+\theta \tilde{x}_{j}^{1}(T, \tau)+\ldots$

$\tilde{y}_{j}=\tilde{y}_{j}^{0}(T, \tau)+\theta \tilde{y}_{j}^{1}(T, \tau)+\ldots$

where $T \sim 1$ is the fast time associated with the blade elastic oscillation, and $\tau=T \theta$ is the slow time, which will retain the small coupling, friction and forcing effects.

The expansions (13) are introduced into the system of motion equations (9), and the following expression is obtained for the first order

$$
\begin{aligned}
\frac{\partial^{2} \tilde{x}_{j}^{0}}{\partial T^{2}}+\tilde{x}_{j}^{0} & =0, \\
\tilde{x}_{j}^{0} & =\bar{F}_{j}\left(\tilde{y}_{j}^{0}\right) .
\end{aligned}
$$

Equation (14) can be straightforwardly solved to give

$\tilde{x}_{j}^{0}=X_{j}(\tau) \mathrm{e}^{\mathrm{i} T}+c . c$.

where, again, complex notation is used because it produces more compact expressions easier to handle than its real counterparts. All airfoils vibrate with the elastic frequency in the fast time scale $T$, while the (complex) amplitude of those oscillations, $X_{j}$, retains the variations in the slow time scale $\tau$. Therefore, at first order, the motion of the blades corresponds simply to the natural oscillation with the blade alone frequency $(\omega=1)$ and with the microslip DOF stuck.

If the disk coupling is retained, the natural frequencies of the TW modes, $\tilde{x}_{j}=A_{m} \mathrm{e}^{\mathrm{i}\left(m \frac{2 \pi}{N} j+\omega_{H t} T\right)}$, are given by the expression

$\omega_{m}=\sqrt{1+2 \frac{k_{c}}{k_{b}}\left(1-\cos \left(m \frac{2 \pi}{N}\right)\right)}$

which is obtained from system (9) just by removing the damping ( $c=0$ ) and the forcing ( $f=0$ ), and assuming the contacts stuck $\left(\tilde{y}_{j}=0\right)$. The TW index $m$ goes from 0 to $N-1$, and the frequency of the TW 


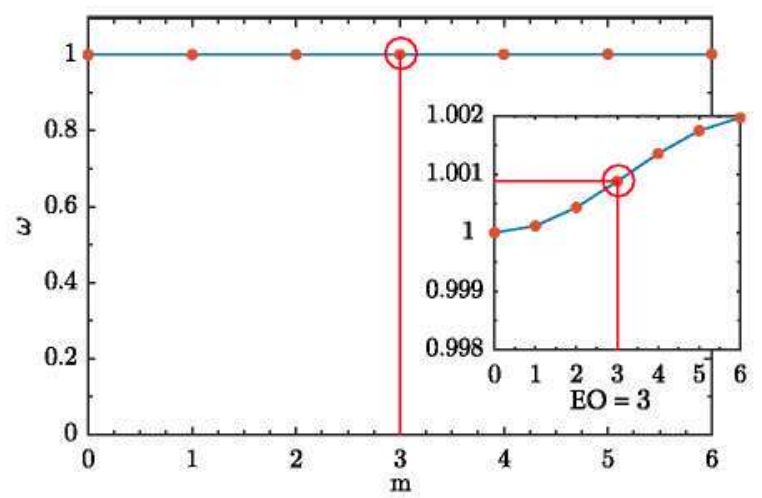

Fig. 3 Tuned natural vibration frequencies of a nearly flat modal family ( $E O$ marks the engine order of the forcing). Inset shows a zoom near $\omega=1$

modes, $\omega_{m}$, grows from 1 to $\sqrt{1+4 \frac{k_{c}}{k_{b}}}$. In the case of small disk coupling considered in this paper, the ratio between blade and coupling stiffness $\left(\frac{k_{c}}{k_{b}}\right)=\theta k$ is very small, and, in first approximation, all mode frequencies are very similar and equal to 1 (blade alone frequency). Figure 3 shows the tuned natural vibration frequencies versus the TW index $m$ for a small value of $\theta=0.001$, and $k=1$. The plot shows an almost horizontal line that corresponds to a typical family of blade-dominated modes.

In the left hand side of the second equation of the system (14) $\tilde{x}_{j}^{0}$ is now determined, and can be employed to compute the first order of the friction DOF displacement $\tilde{y}_{j}^{0}$. A real periodic function in $T$ is obtained, which can be expressed as a Fourier series

$\tilde{y}_{j}^{0}=P\left(T, X_{j}\right)=\sum_{k=1}^{\infty} P_{k}\left(X_{j}\right) \mathrm{e}^{\mathrm{i} k T}+$ c.c.

Note that this is a periodic function with the same $2 \pi$ period as the blades (15), but now all time harmonics are produced after solving the second equation of the system (14) to obtain $\tilde{y}_{j}^{0}$ because of the nonlinear friction force.

The next order approximation is obtained taking the expressions (13) into Eq. (9) and retaining the next order terms

$$
\begin{aligned}
& \frac{\partial^{2} \tilde{x}_{j}^{1}}{\partial T^{2}}+2 \frac{\partial^{2} \tilde{x}_{j}^{0}}{\partial T \partial \tau}+\tilde{x}_{j}^{1}-\tilde{y}_{j}^{0}+k\left(2 \tilde{x}_{j}^{0}-\tilde{x}_{j+1}^{0}-\tilde{x}_{j-1}^{0}\right) \\
& \quad+c \frac{\partial \tilde{x}_{j}^{0}}{\partial T}=f \mathrm{e}^{i \tilde{\omega} T+\mathrm{i} E O \frac{2 \pi}{N} j}+\text { c.c. }
\end{aligned}
$$

Now, making use of the expressions for $\tilde{x}_{j}^{0}(14)$ and $\tilde{y}_{j}^{0}$ (17), the preceding Eq. (18) can be re-formulated as

$$
\begin{aligned}
& \frac{\partial^{2} \tilde{x}_{j}^{1}}{\partial T^{2}}+\tilde{x}_{j}^{1}=-2 \mathrm{i} \frac{\partial X_{j}}{\partial \tau} \mathrm{e}^{\mathrm{i} T}+\sum_{k=1}^{\infty} P_{k}\left(X_{j}\right) \mathrm{e}^{\mathrm{i} k T} \\
& -\left(k\left(2 X_{j}-X_{j+1}-X_{j-1}\right)+\mathrm{i} c X_{j}\right) \mathrm{e}^{\mathrm{i} T} \\
& +f \mathrm{e}^{\mathrm{i} T} \mathrm{e}^{i \Delta \omega \theta T+\mathrm{i} E O \frac{2 \pi}{N} j}+\text { c.c. }
\end{aligned}
$$

where the forcing frequency has also been expanded as $\tilde{\omega}=1+\theta \Delta \omega$, to describe a forcing near the resonant frequency $\omega_{0}=1$.

A bounded evolution for $\tilde{x}_{j}^{1}(T, \tau)$ is only possible if there are no terms proportional to $\mathrm{e}^{\mathrm{i} T}$ in the right hand side of the previous expression, see $[1,4]$. This constraint provides the evolution equation for the blade amplitudes $X_{j}$ in the slow time scale $\tau$

$$
\begin{aligned}
2 \mathrm{i} \frac{\partial X_{j}}{\partial \tau}= & P_{1}\left(X_{j}\right)-k\left(2 X_{j}-X_{j+1}-X_{j-1}\right) \\
& -\mathrm{i} c X_{j}+f \mathrm{e}^{i \Delta \omega \tau+\mathrm{i} E O \frac{2 \pi}{N} j}+\text { c.c. }
\end{aligned}
$$

The first Fourier coefficient $P_{1}$ of the cycle of $\tilde{y}_{j}^{0}$ represents the work of the friction forces, and contains all the relevant information about the nonlinear friction loop. It can be expressed introducing the nonlinear friction coefficient $Q\left(\left|X_{j}\right|\right)$

$P_{1}\left(X_{j}\right)=Q\left(\left|X_{j}\right|\right) X_{j}$,

as the cycle of $\tilde{y}_{j}^{0}$ is in phase with that of $\tilde{x}_{j}^{0}$, see Eq. (14). It depends only on the friction model that is used, and comprises all the effects from the nonlinear friction forces: its real part produces a nonlinear correction of the frequency, and the imaginary part gives a nonlinear damping that grows with the vibration amplitude (see Fig. 4).

The asymptotically reduced order model can be finally formulated as

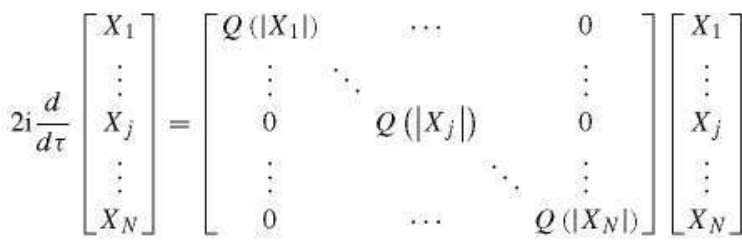

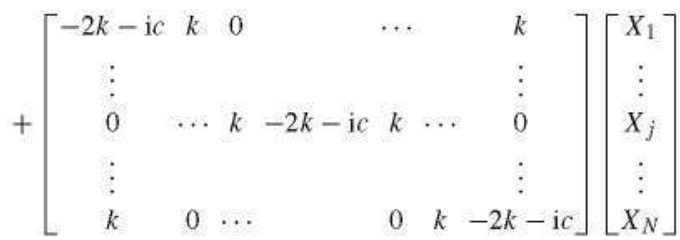




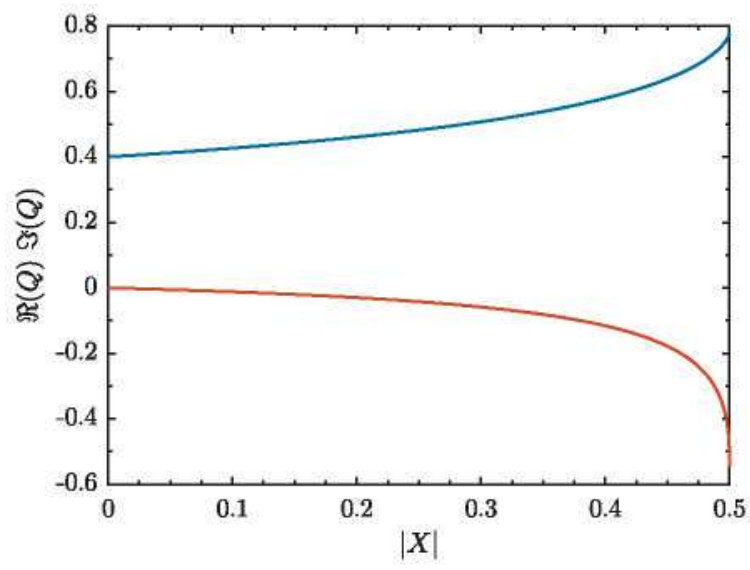

Fig. 4 Friction coefficient $Q(|X|)$ for the Olofsson microslip model, real part (blue) and imaginary part (red)

$$
+f\left[\begin{array}{c}
\mathrm{e}^{\mathrm{i} E O \frac{2 \pi}{N}} \\
\vdots \\
\mathrm{e}^{\mathrm{i} E O \frac{2 \pi}{N} j} \\
\vdots \\
\mathrm{e}^{\mathrm{i} E O 2 \pi}
\end{array}\right] \mathrm{e}^{\mathrm{i} \Delta \omega \tau},
$$

where $X_{j}$ stands for the complex amplitude of the displacement of blade $j$. The first matrix corresponds to the effect of the friction and it is a diagonal matrix (the fiction of each blade has an effect only on the vibration of this blade). All the elastic and linear damping effects are included in the second matrix. Note that the terms $k,-2 k, k$ correspond to the first-order elastic correction of the frequencies of the nearly flat modal family that we are considering, which includes the coupling with the adjacent blades. A different mass spring model would produce a similar set of asymptotic equations but with different elastic correction terms. The last term accounts for the external forcing, and $\Delta \omega$ is the deviation of the forcing frequency from the blade alone resonant frequency. The numerical integration of this system requires very low CPU cost because the fast elastic vibration time has been completely filtered out.

The final blade oscillation, in the tuned case, is composed of just one TW, and, therefore, the blade displacement $X_{j}$ can be written as

$$
X_{j}=A \mathrm{e}^{\mathrm{i} E O \frac{2 \pi}{N} j+\mathrm{i} \Delta \omega \tau}
$$

i.e., all the blades oscillate with the same frequency $(\Delta \omega)$ and same vibration amplitude $(A)$ but with different phases $\left(E O \frac{2 \pi}{N} j\right)$. Consequently, the previous reduced order model can be simplified even further to give:

$$
\begin{aligned}
& 2 \mathrm{i} A\left[\begin{array}{c}
\mathrm{e}^{\mathrm{i} E O \frac{2 \pi}{N}} \\
\vdots \\
\mathrm{e}^{\mathrm{i} E O \frac{2 \pi}{N} j} \\
\vdots \\
\mathrm{e}^{\mathrm{i} E O 2 \pi}
\end{array}\right] \frac{d}{d \tau} \mathrm{e}^{\mathrm{i} \Delta \omega \tau} \\
& =A\left[\begin{array}{ccccc}
Q(|A|) & & \cdots & & 0 \\
\vdots & \ddots & & & \vdots \\
0 & & Q(|A|) & 0 \\
\vdots & & & \ddots & \vdots \\
0 & & \cdots & & Q(|A|)
\end{array}\right]\left[\begin{array}{c}
\mathrm{e}^{\mathrm{i} E O \frac{2 \pi}{N}} \\
\vdots \\
\mathrm{e}^{\mathrm{i} E O \frac{2 \pi}{N} j} \\
\vdots \\
\mathrm{e}^{\mathrm{i} E O 2 \pi}
\end{array}\right] \mathrm{e}^{\mathrm{i} \Delta \omega \tau}
\end{aligned}
$$

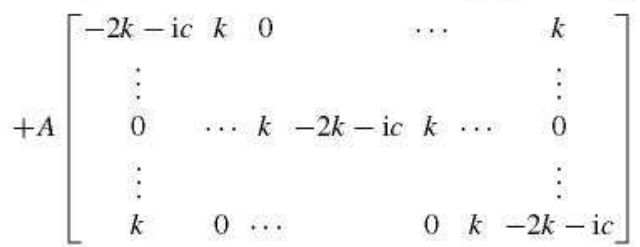

$$
\begin{aligned}
& {\left[\begin{array}{c}
\mathrm{e}^{\mathrm{i} E O \frac{2 \pi}{N}} \\
\vdots \\
\mathrm{e}^{\mathrm{i} E O \frac{2 \pi}{N} j} \\
\vdots \\
\mathrm{e}^{\mathrm{i} E O 2 \pi}
\end{array}\right] \mathrm{e}^{\mathrm{i} \Delta \omega \tau}+f\left[\begin{array}{c}
\mathrm{e}^{\mathrm{i} E O \frac{2 \pi}{N}} \\
\vdots \\
\mathrm{e}^{\mathrm{i} E O \frac{2 \pi}{N} j} \\
\vdots \\
\mathrm{e}^{\mathrm{i} E O 2 \pi}
\end{array}\right] \mathrm{e}^{\mathrm{i} \Delta \omega \tau},}
\end{aligned}
$$

and taking into account that the second matrix-vector multiplication on the right hand side is developed as

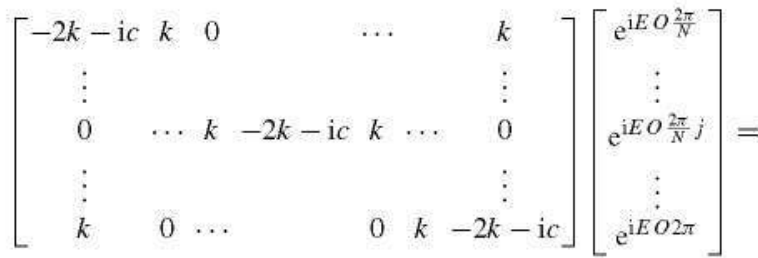

$$
\begin{aligned}
& =\left[2 k\left(\cos \left(E O \frac{2 \pi}{N}\right)-1\right)-\mathrm{i} c\right]\left[\begin{array}{c}
\mathrm{e}^{\mathrm{i} E O \frac{2 \pi}{N}} \\
\vdots \\
\mathrm{e}^{\mathrm{i} E O \frac{2 \pi}{N} j} \\
\vdots \\
\mathrm{e}^{\mathrm{i} E O 2 \pi}
\end{array}\right],
\end{aligned}
$$

the reduced order model can be condensed to a single equation 


$$
\begin{aligned}
& {\left[2 \Delta \omega A+Q(|A|) A+2 k\left(\cos \left(E O \frac{2 \pi}{N}\right)-1\right) A-\mathrm{i} C A+f\right]} \\
& {\left[\begin{array}{c}
\mathrm{e}^{\mathrm{i} E O \frac{2 \pi}{N}} \\
\vdots \\
\mathrm{e}^{\mathrm{i} E O \frac{2 \pi}{N} j} \\
\vdots \\
\mathrm{e}^{\mathrm{i} E O 2 \pi}
\end{array}\right] \mathrm{e}^{\mathrm{i} \Delta \omega \tau}=0 .}
\end{aligned}
$$

Therefore, the calculation of the blade amplitude $A$ of the tuned TWs with the forcing dissipated by the nonlinear friction forces can be obtained solving just one complex equation:

$$
\begin{aligned}
& {\left[2 \Delta \omega+Q(|A|)+2 k\left(\cos \left(E O \frac{2 \pi}{N}\right)-1\right)-\mathrm{i} c\right]} \\
& A+f=0
\end{aligned}
$$

Several simulations of the full mass-spring model of Eq. (9) have been performed with the objective of validating the accuracy of the asymptotic model obtained in this section. Numerical calculations have been performed in MATLAB, employing a constant time step 4 th order Runge-Kutta scheme. The integration of the mass-spring system is very expensive because the time step has to be small enough to solve the short friction time scale. Similarly, the integration time has to be large enough to allow the system to execute many elastic oscillation periods until the blade displacement reaches a stationary limit cycle oscillation.

In the simulations, the number of blades is set to $N=$ 13, the engine order to $E O=3$, the forcing amplitude to $f=0.04$, the stiffness ratio to $k=1$, the mass friction parameter to $\gamma=1$, and the material damping $c=0.05$. Regarding the blade to friction stiffness ratio, it is reduced to $\theta=0.1,0.01$, and 0.001 to approach the asymptotic limit $\theta \ll 1$.

The frequency sweeps corresponds to sweeping in $\Delta \omega$, which is the deviation of the forcing frequency from the $0^{\text {th }}$-order resonance: $\omega=1+\theta \Delta \omega$. They are plotted in Fig. 5, showing a clear convergence of the results as the value of $\theta$ is decreased. In fact, the values for the case $\theta=0.01$ are almost indistinguishable from the ones of the case $\theta=0.001$. The asymptotic results from the simplified equation (26) are also depicted, exhibiting an excellent agreement with the cases with $\theta=0.01$ and $\theta=0.001$.

Figure 6 displays the TW content of the solution at the maximum value reached when sweeping in frequency. As it was predicted by the asymptotic model, in

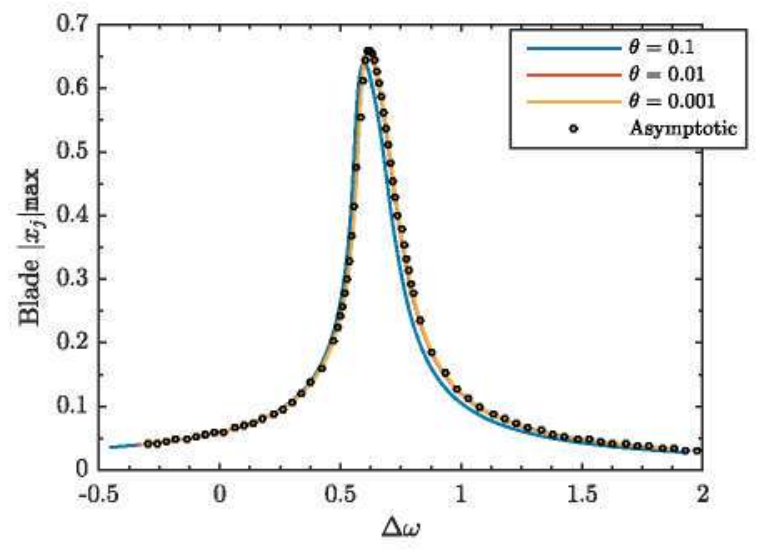

Fig. 5 Forced response of the tuned system
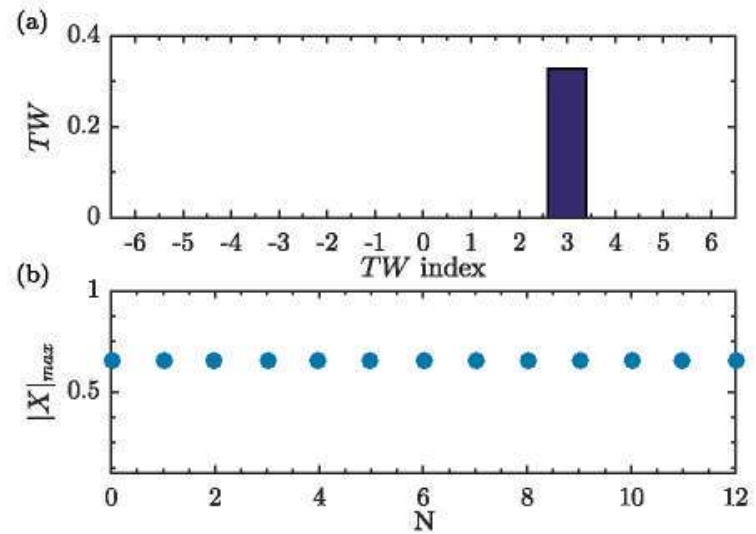

Fig. 6 Amplitude of the travelling wave (a) and displacement of the blade (b), located where maximum forced response is reached (see Fig. 5)

this tuned case the solution is approximately composed of a single TW with index 3 . This result corresponds to the TW directly excited by the external forcing. There are other TWs in the response of the system which are produced by the nonlinear friction forces, but they have very small amplitudes (below $10^{-4}$ ) and cannot be seen in the plot.

The asymptotic model for the tuned TW amplitudes case (26) is the same as that obtained in [7]. But the analysis presented in this section has two main differences: (i) a complete family of modes with similar frequencies is considered here while in [7] only two isolated modes were included in the analysis, and (ii) the derivation in [7] was performed in TW formulation and here we use the blade displacement amplitude, which can be more easily generalized to the mistuned case. 


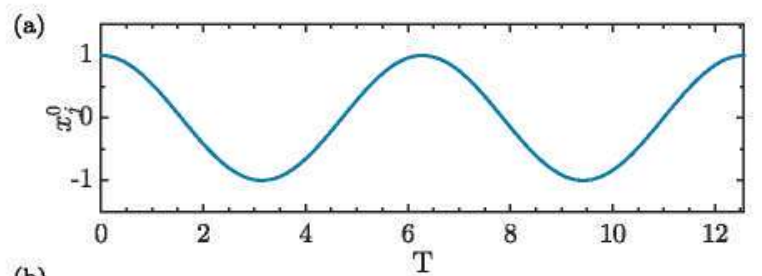

(b)

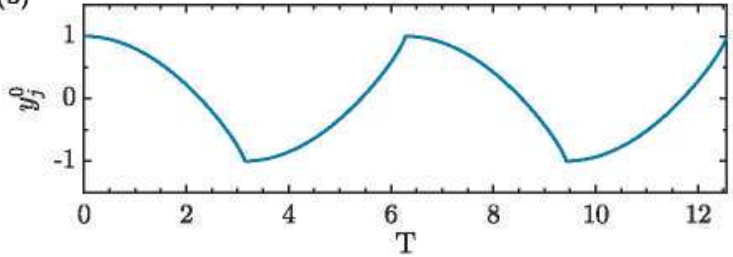

Fig. 7 Time evolution of blade displacement (a) and microslip displacement (b)

Note that, despite of the nonlinear character of the friction forces, the motion of the blades is practically harmonic in time, as given by eq. (15). However, the friction DOFs oscillation obtained in eq. (17) is completely nonlinear. The asymptotic method has the ability of retaining all harmonics of the nonlinear cycle of the friction DOFs (see Fig. 7). This is a clear advantage with respect to other methods for computing the nonlinear forced response of blade disks like the Harmonic

\section{Mistuned response}

The effect of mistuning is accounted for adding a small variation to the stiffness of the blades, $\theta k_{j}$, therefore the term $k_{b}$ (identical for all blades in the tuned case) becomes now $k_{b j}=k_{b}\left(1+\theta k_{j}\right)$ (different for each blade when mistuned is considered). Mistuning is a small effect and is scaled with the small parameter $\theta$. The resulting nondimensional equations for the massspring model with mistuning included are given by

$$
\begin{aligned}
& \frac{d^{2} \tilde{x}_{j}}{d T^{2}}+\left(1+\theta k_{j}\right)\left(\tilde{x}_{j}-\theta \tilde{y}_{j}\right) \\
& \quad+\theta k\left(2 \tilde{x}_{j}-\tilde{x}_{j+1}-\tilde{x}_{j-1}\right)+\theta c \frac{d \tilde{x}_{j}}{d T}= \\
& \quad=\theta f \mathrm{e}^{i \tilde{\omega} T+\mathrm{i} E O \frac{2 \pi}{N} j}+c . c . \\
& \theta \gamma \frac{d^{2} \tilde{y}_{j}}{d T^{2}}+\left(\theta \tilde{y}_{j}-\tilde{x}_{j}\right)\left(1+\theta k_{j}\right) \\
& \quad+\tilde{F}_{f}\left(\tilde{y}_{j}\right)=0 \text { for } j=1, \ldots, N .
\end{aligned}
$$

The application of the multiple scale method is completely similar to that in the previous section, and the resulting asymptotically reduced order model just shows some extra terms due to mistuning (blade frequency variations) in the diagonal of the last matrix:

$$
\begin{aligned}
& 2 \mathrm{i} \frac{d}{d \tau}\left[\begin{array}{c}
X_{1} \\
\vdots \\
X_{j} \\
\vdots \\
X_{N}
\end{array}\right]=\left[\begin{array}{ccccc}
Q\left(\left|X_{1}\right|\right) & & \cdots & & 0 \\
\vdots & \ddots & & & \vdots \\
0 & & Q\left(\left|X_{j}\right|\right) & 0 \\
\vdots & & & \ddots & \vdots \\
0 & & \cdots & & Q\left(\left|X_{N}\right|\right)
\end{array}\right]\left[\begin{array}{c}
X_{1} \\
\vdots \\
X_{j} \\
\vdots \\
X_{N}
\end{array}\right]
\end{aligned}
$$

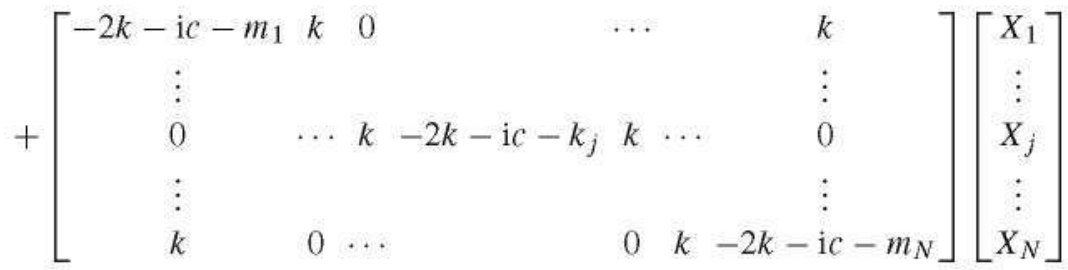

$$
\begin{aligned}
& +f\left[\begin{array}{c}
\mathrm{e}^{\mathrm{i} E O \frac{2 \pi}{N}} \\
\vdots \\
\mathrm{e}^{\mathrm{i} E O \frac{2 \pi}{N} j} \\
\vdots \\
\mathrm{e}^{\mathrm{i} E O 2 \pi}
\end{array}\right] \mathrm{e}^{\mathrm{i} \Delta \omega \tau}
\end{aligned}
$$

Balance Method [11] that retain only a few harmonics for the friction DOFs.
As it was done in the previous section, we can look for the final limit cycle oscillation state, with a blade 
displacement $X_{j}$ of the form

$X_{j}=A_{j} \mathrm{e}^{\mathrm{i} \Delta \omega t}$

where all the blades oscillate with the same frequency $(\Delta \omega)$. But, in this case, due to mistuning effects, the blade movement can be now composed of many different travelling waves. Therefore, the vibration amplitude $\left(A_{j}\right)$ is now different for each blade.

Introducing the preceding expression in the asymptotic mistuned model (28), the following system is obtained small ratio of blade and coupling stiffness, and small mistuning effects, and compared with the results from the asymptotic model. Figure 8 displays the response of the mass-spring system for different small values of the parameter $\theta(\theta=0.1, \theta=0.0 \mathrm{~L}$ and $\theta=0.00 \mathrm{l})$ and a random mistuning pattern (all the remaining parameters are set as in the tuned simulations of the previous section). There is again a clear convergence towards the asymptotic results. As it happened in the tuned case, the plot shows that the cases $\theta=0.01$ and $\theta=0.001$ are almost identical. The usual effect of trequency split-

$$
\begin{aligned}
& 2 \mathrm{i}\left[\begin{array}{c}
A_{1} \\
\vdots \\
A_{j} \\
\vdots \\
A_{N}
\end{array}\right] \frac{d}{d \tau} \mathrm{e}^{\mathrm{i} \Delta \omega \tau}=\left[\begin{array}{ccccc}
Q\left(\left|A_{1}\right|\right) & & \cdots & & 0 \\
\vdots & \ddots & & & \vdots \\
0 & & Q\left(\left|A_{j}\right|\right) & & 0 \\
\vdots & & & \ddots & \vdots \\
0 & & \cdots & & Q\left(\left|A_{N}\right|\right)
\end{array}\right]\left[\begin{array}{c}
A_{1} \\
\vdots \\
A_{j} \\
\vdots \\
A_{N}
\end{array}\right] \mathrm{e}^{\mathrm{i} \Delta \omega \tau}
\end{aligned}
$$

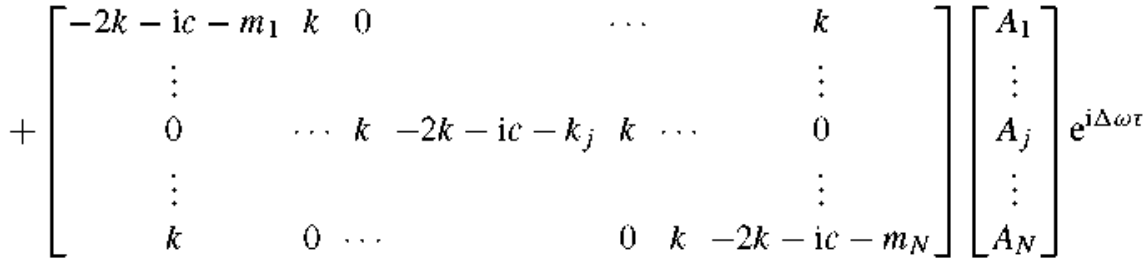

$$
\begin{aligned}
& +f\left[\begin{array}{c}
\mathrm{e}^{\mathrm{i} E O \frac{2 \pi}{N}} \\
\vdots \\
\mathrm{e}^{\mathrm{i} E O \frac{2 \pi}{N} j} \\
\vdots \\
\mathrm{e}^{\mathrm{i} E O 2 \pi}
\end{array}\right] \mathrm{e}^{\mathrm{i} \Delta \omega \tau}
\end{aligned}
$$

which can be rearranged as

$$
\left[2 \Delta \omega \mathbf{I}+\operatorname{diag}\left(Q\left(\left|A_{j}\right|\right)\right)+\mathbf{K}\right]\left[\begin{array}{c}
A_{1} \\
\vdots \\
A_{j} \\
\vdots \\
A_{N}
\end{array}\right]+f\left[\begin{array}{c}
\mathrm{e}^{\mathrm{i} E O \frac{2 \pi}{N}} \\
\vdots \\
\mathrm{e}^{\mathrm{i} E O \frac{2 \pi}{N} j} \\
\vdots \\
\mathrm{e}^{\mathrm{i} E O 2 \pi}
\end{array}\right]=0
$$

where $\mathbf{K}$ is the matrix with the stiffness, linear damping and mistuning effects, $\mathbf{I}$ is the identity matrix, and diag $\left(Q\left(\left|A_{j}\right|\right)\right)$ stands for a diagonal matrix with the vector $Q\left(\left|A_{j}\right|\right)$ in the diagonal. This is a nonlinear system of $N$ algebraic equations that allows for a very fast calculation of the blade amplitudes $A_{j}$.

Once more, the dynamics of the mass-spring system of Eq. (27) is analyzed in the limit of small forcing, small damping (nonlinear friction and material), ting of the maximum response due to mistuning can be seen, together with an amplification (above $25 \%$ ) of the response compared to the tuned case, see Fig. 5.

Now, we proceed to integrate the system of equations (28) with the aim of examining the combined effect of mistuning and microslip friction, employing the following parameter values: $N=13, E O=3$, $f=0.04, k=1, \gamma=1, c=0.05$. The mistuning pattern is same random distribution of the simulations of the previous section, normalized to have $\max _{j}\left|k_{j}\right|=1$ and then scaled by a factor that grows from $0 \%$ (tuned case) to $40 \%, 80 \%, 120 \%, 200 \%$ and $300 \%$. Note that the actual value of applied mistuning is $\theta k_{j}$, still small as it is multiplied by the small parameter $\theta \ll 1$. Figure 9 shows the value of this random pattern for each blade. 


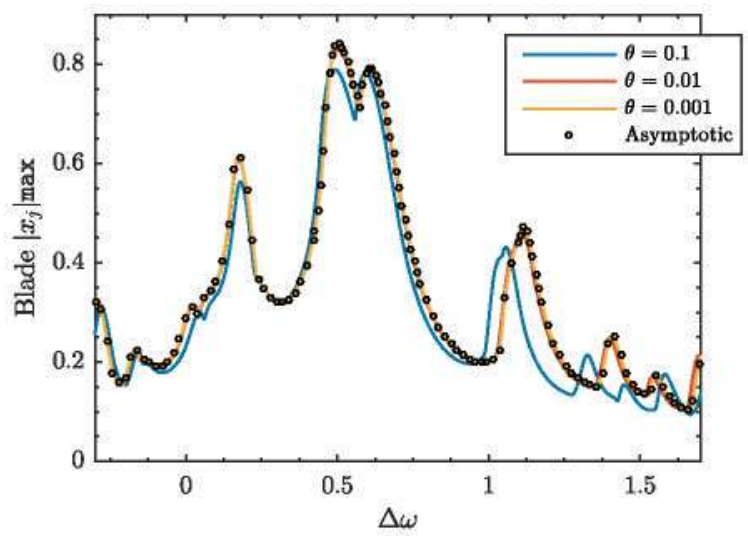

Fig. 8 Frequency sweeps of the mistuned system

Figure 10 depicts the resulting frequency sweeps for the previous values of mistuning intensities. The response is similar to the case of constant linear damping found in [6]: mistuning produces an evident amplification, reaching a $28 \%$ of gain near for the case of $80 \%$ mistuning. Although, in the present case, the maximum amplitude does not decay back to the tuned value, instead it remains almost constant for large mistuning values. If the mistuned considered is a fraction $(40 \%$ f.i.) of the one used as reference, the maximum response is isolated near the resonance frequency (central large split peaks). However, as mistuning is increased $(300 \%)$, the response peaks spreads over all the range of frequencies simulated (several peaks with almost same value distributed over the frequency domain).

Now, selecting the maximum response point represented in Fig. 10 for the mistuning cases, 40\%, $120 \%$, and $200 \%$, the corresponding TW spectrum and blade displacement of the system are represented in Figs. 11, 12, and 13, respectively. A comparison can be done considering the tuned case illustrated in Fig. 6 . The solution now is notably different: it is no longer just one TW with index 3 , but it shows another TW with index -3 . The rest of TWs appears with less intensity (remaining below $10^{-1}$ in Fig. 11) to fill the spectrum as the mistuning is increased. The results are also significantly different from the case with a ratio between the blade and coupling stiffness $\left(\frac{k_{c}}{k_{b}}\right)$ not small (isolated mode forcing, see [7]). In that case, the response is only composed of the TW with index 3 and -3 , and the all other TWs are practically not excited.

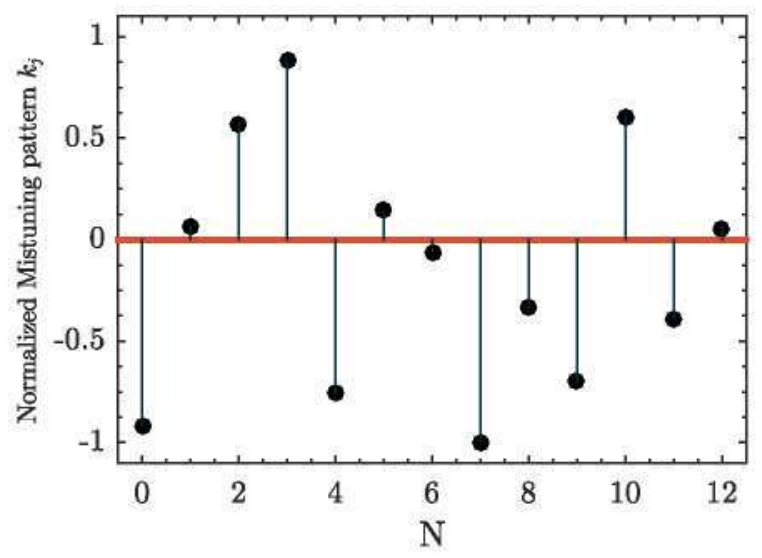

Fig. 9 Random mistuning pattern of each blade $\left(k_{j}\right)$

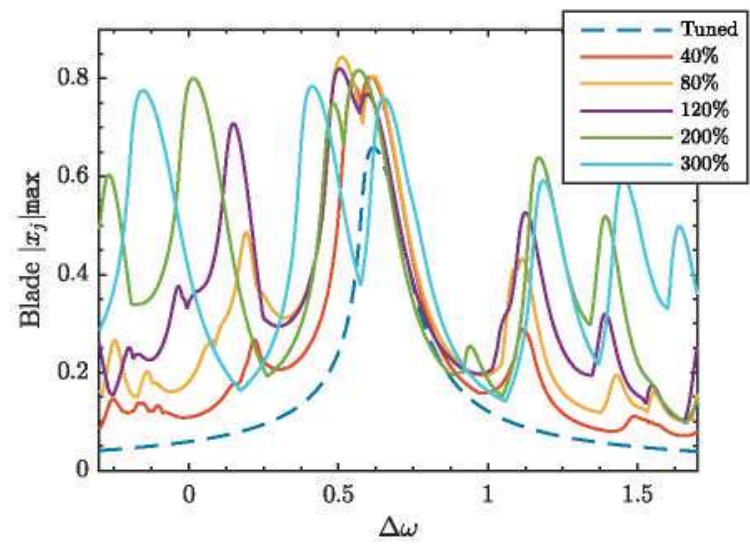

Fig. 10 Forced response of the system for $0 \%$ (tuned case), $40 \%$, $80 \%, 120 \%, 200 \%$ and $300 \%$ mistuning
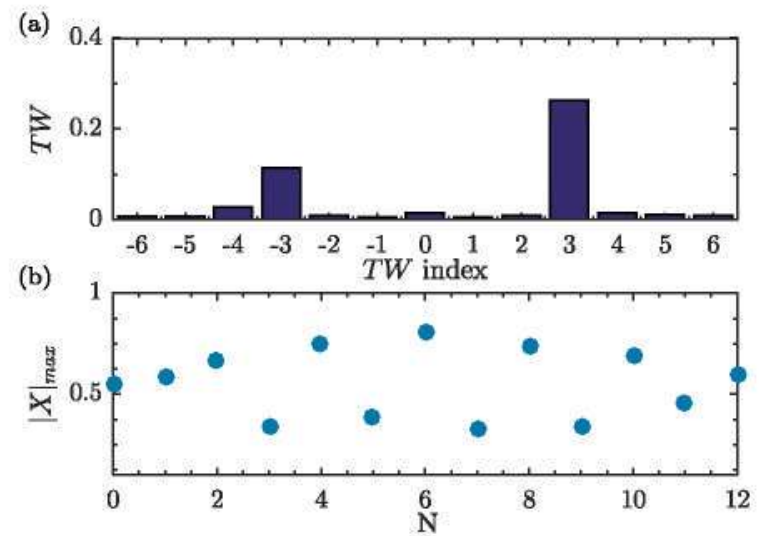

Fig. 11 Amplitude of the travelling wave (a) and displacement of the blade (b), located where maximum forced response is reached at $40 \%$ of mistuning (see Fig. 10) 

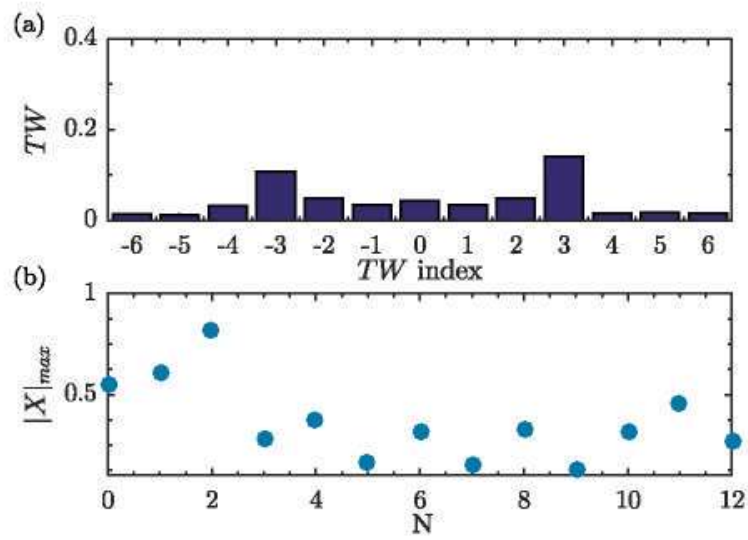

Fig. 12 Amplitude of the travelling wave (a) and displacement of the blade (b), located where maximum forced response is reached at $120 \%$ of mistuning (see Fig. 10)

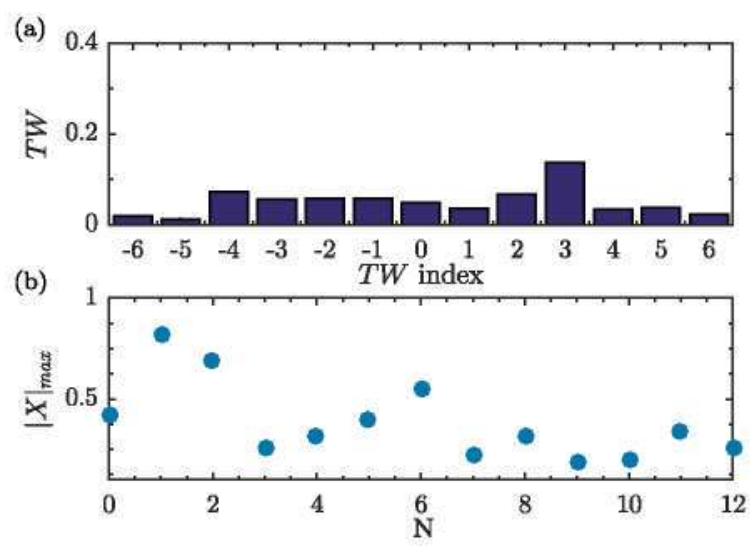

Fig. 13 Amplitude of the travelling wave (a) and displacement of the blade (b), located where maximum forced response is reached at $200 \%$ of mistuning (see Fig. 10)

Finally, the forced response amplification is presented as function of the scale factor of mistuning in Fig. 14. It can be observed that the maximum amplification appears around $100 \%$ of the mistuned analyzed, corresponding to a gain of approximately $28 \%$. In addition, the maximum amplitude appears to be maintained for larger mistuning values than in the linear damping case.

\section{Validation against experimental data}

There are not many experiments available in the literature with detailed measurements of the forced response vibration of a bladed disk saturated by nonlinear friction effects. In [3] a realistic low pressure turbine (LPT)

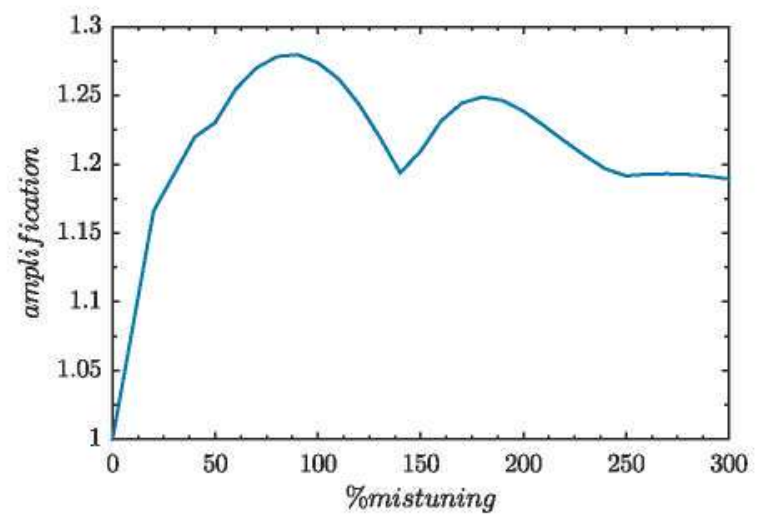

Fig. 14 Forced response amplification variation with mistuning

rotor with $\mathrm{N}=146$ blades was tested at the in-vacuum, rotating rig STARGATE facility at Avio Aero. The rotor had a blade-dominated, nearly flat modal family (see Fig. 5 of [3]), that was excited by a TW forcing implemented using magnets mounted on a second wheel that rotated independently from the LPT rotor. The left plot of Fig. 15 displays the Blade Tip Timing (BTT) measured vibration amplitude of one of the rotor blades as a function of the forcing frequency. The nonlinear effect of the friction dissipation produced at the fir-tree can be clearly appreciated in the non-symmetric resonant response with respect to the maximum vibration amplitude (linear damping would produce a symmetric response). The asymptotic expression for the resonance peak from Eq. (26) gives a quite good fit of the experimental data, see right plot of Fig. 15, showing a similar non-symmetric amplitude dependence on the forcing frequency (the coefficients in Eq. (26) have been adjusted to qualitatively reproduce the experimental data since there is not enough information in [3] for a quantitative comparison).

This indicates that the asymptotic model, despite of its simplicity (1 DOF per sector), can describe the main mechanisms involved in the nonlinear friction saturation of the forced response of a realistic LPT rotor. Note also that the width of the resonance peak is small compared to the resonant frequency itself (less than 5\% for the half amplitude width in Fig. 15). This narrow peak implies that the time signal of the blade displacement is composed of a given frequency with a slow time modulation, as in expression (15). Therefore, this strongly suggests that a multiple scale method can be applied to obtain a simplified description of the problem. 

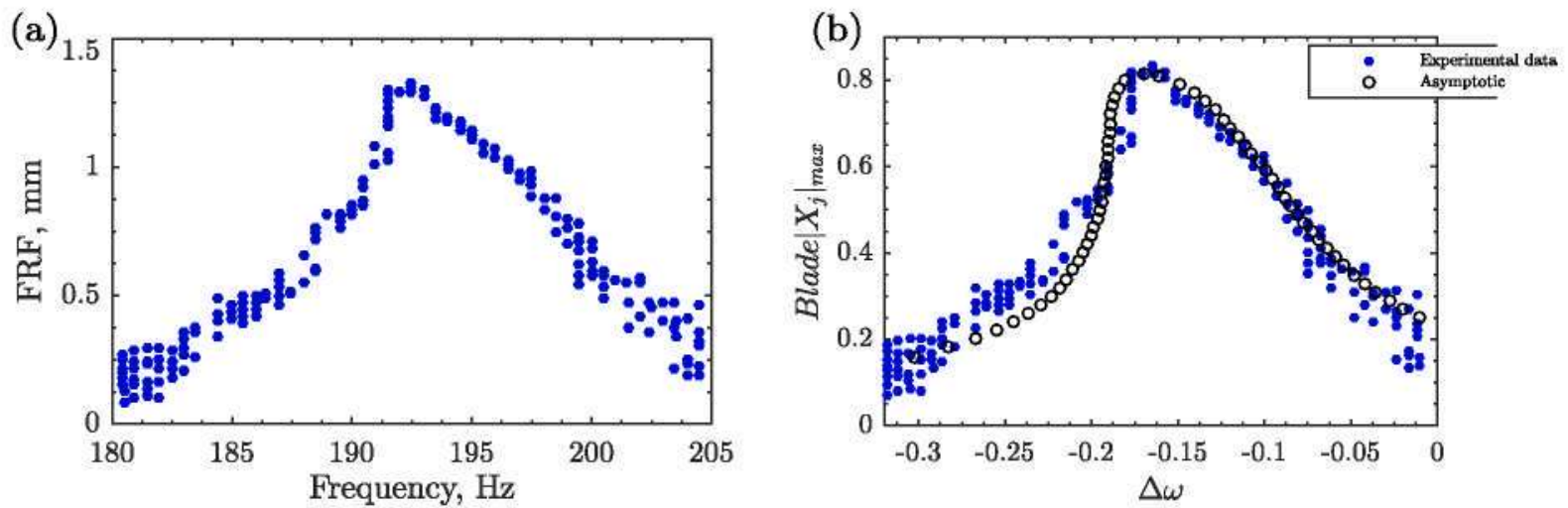

Fig. 15 Left: Blade vibration measurements (from [3]). Right: Asymptotic model results added (circles)

\section{Conclusions}

A mass-spring model is used to represent a mistuned bladed disk with nonlinear friction effects (in the microslip regime) at the blade/disk contact interfaces. The system has a family of blade-dominated modes with very similar vibrations frequencies that is excited by the external forcing. The method of multiple scales is used to simplify the problem and derive asymptotically reduced models that are validated in the tuned and mistuned case.

The asymptotic simplification is based on the fact that the ratios of blade stiffness to the microslip friction stiffness $(\theta)$, to the blade coupling stiffness $(\theta k)$, and to the stiffness mistuning variation $\left(\theta k_{j}\right)$, are all typically small in most realistic configurations. All these small effects are developed in a much slower time scale than that of blade elastic vibration, and are captured in the asymptotic reduced models derived in this paper in the limit $\theta \ll 1$.

The resulting asymptotic models are very simple, and the accuracy of its solutions has been checked against simulations of the complete mass-spring model. The results show an excellent agreement for values of $\theta=0.01$ and below.

The computation of the final amplitude of the response of the system in the asymptotic model can be reduced to solving a single equation in the tuned case, and a system of $N$ nonlinear equations in the mistuned case. This drastic reduction is not possible in the original mass-spring system because of the complex nonlinear behaviour of the friction forces whose magnitude depends on the previous loading-unloading switching point.
The simulation of the original mass-spring model demands to integrate a system of $2 N$ differential equations with stiff terms coming from the nonlinear friction. Large integration times with small time step are required in order to properly account for all small effects and to reach the final periodic state. In a desktop workstation, the frequency sweep corresponding to the $\theta=0.001$ case is performed in 90 hours. On the other hand, the asymptotic model needs only 4 seconds. This huge speed-up would allow to conduct parametric studies, statistical analysis or any other investigation involving a large number of numerical simulations, which are practically not manageable with the complete mass-spring model.

The asymptotic model reveals also the mechanism of action of the friction and mistuning effects: (i) in first approximation the system response is just a general combination of the linear tuned modes computed with the contact interfaces stuck, (ii) the blade motion induces forces in the contact interfaces that are used to obtain the small motion of the friction DOFs, and (iii) this small motion produces a small (nonlinear) damping of the blade oscillation that set-in together with the effect of mistuning, in a time scale much longer than elastic vibration period of the blades.

A comparison to available experimental data on the forced response vibration of a bladed disk with nonlinear friction effects has been also presented. It shows a good qualitative agreement, indicating that this simple asymptotic model can be employed to capture the main mechanisms involved in the forced response vibration of a realistic LPT rotor with nonlinear damping effects coming from the friction forces at the blade-disk contact interfaces. 
In conclusion, the multiple scale method has been applied to a very reduced model with just 2 DOF per sector, with the idea in mind of presenting a step by step explanation of the application of this methodology to the simplest possible bladed disk model showing a blade-dominated modal family. The applicability of the multiple scale method is based on the presence of effects with very different order of magnitude (friction, damping, blade coupling, and mistuning much smaller than the elastic effects), which is precisely the situation in many aeroelasticity turbomachinery problems. Even in the case of a Integrally Bladed Rotor made of one single metal piece (i.e. with no contact interfaces), without nonlinear friction forces, this methodology could be used to obtain a simplified description of other small effects responsible of the saturation of the blade vibration amplitude, such as nonlinear geometric elastic terms or aerodynamic damping. In consequence, this formulation can be applied in a similar way to more detailed FEM descriptions of bladed disks, therefore future research activity will be focused on this problem.

Acknowledgements This work has been supported by the Spanish Ministerio de Ciencia, Innovación y Universidades under Grant DPI2017-84700-R.

\section{Compliance with ethical standards}

Conflict of interest The authors declare that they have no conflict of interest.

\section{References}

1. Bender, C.M., Orszag, S.A.: Advanced Mathematical Methods for Scientists and Engineers: I: Asymptotic Methods and Perturbation Theory. Springer, New York (1999). https://doi. org/10.1007/978-1-4757-3069-2

2. Corral, R., Gallardo, J.M.: A methodology for the vibration amplitude prediction of self-excited rotors based on dimensional analysis. In: Proceedings of ASME Turbo Expo 2016: Turbomachinery Technical Conference and Exposition, ASME, GT2006-90668, pp 1101-1113 (2016) https:// doi.org/10.1115/GT2006-90668
3. Corral, R., Beloki, J., Calza, P., Elliott, R.: Flutter generation and control using mistuning in a turbine rotating rig. AIAA J. 57(2), 782-795 (2019). https://doi.org/10.2514/1.J056943

4. Kevorkian, J., Cole, J.: Multiple Scale and Singular Perturbation Methods, Applied Mathematical Sciences, vol 114. Springer, New York (1996). https://doi.org/10.1007/ 978-1-4612-3968-0

5. Krack, M., Salles, L., Thouverez, F.: Vibration prediction of bladed disks coupled by friction joints. Arch. Comput. Methods Eng. 24, 589-636 (2017). https://doi.org/10.1007/ s11831-016-9183-2

6. Martel, C., Corral, R.: Asymptotic description of maximum mistuning amplification of bladed disk forced response. J. Eng. Gas Turbin. Power 131(2), 022506 (2009), https://doi. org $/ 10.1115 / 1.2968868$

7. Martel, C., Martin, J.A.: Asymptotic description of forced response vibration saturation by friction forces. J. Eng. Gas Turbines Power 142(2), 011022:1-8 (2020). https://doi.org/ 10.1115/1.4044934

8. Martel, C., Corral, R., Ivaturi, R.: Flutter amplitude saturation by nonlinear friction forces: Reduced model verification. J. Turbomach. 137(4), 041004:1-8 (2014). https://doi. org/10.1115/1.4028443

9. Olofsson, U.: Cyclic microslip under unlubricated conditions. Tribol. Int. 28(4), 207-217 (1995). https://doi.org/10. 1016/0301-679X28942900001-7

10. Petrov, E.P., Ewins, D.J.: Analytical formulation of friction interface elements for analysis of nonlinear multi-harmonic vibrations of bladed disks. J. Turbomach. 125(2), 364-371 (2003). https://doi.org/10.1115/1.1539868

11. Petrov, E.P., Ewins, D.J.: State-of-the-art dynamic analysis for non-linear gas turbine structures. Proc. Inst. Mech. Eng. Part G: J. Aerosp. Eng. 218(3), 199-211 (2004). https://doi. org/10.1243/0954410041872906

12. Yang, B., Menq, C.: Characterization of $3 \mathrm{~d}$ contact kinematics and prediction of resonant response of structures having a 3d frictional constraint. J. Sound Vib. 217(5), 909-925 (1998). https://doi.org/10.1006/jsvi.1998.1802

13. Yang, B., Chu, M., Menq, C.: Stick-slip-separation analysis and non-linear stiffnes and damping characterization of friction contacts having variable normal load. J. Sound Vib. 210(4), 461-481 (1998). https://doi.org/10.1006/jsvi.1997. 1305

Publisher's Note Springer Nature remains neutral with regard to jurisdictional claims in published maps and institutional affiliations. 